\title{
Automatic Facial Spots and Acnes Detection System
}

\section{Chuan-Yu Chang, Heng-Yi Liao}

Department of Computer Science and Information Engineering, National Yunlin University of Science and Technology, Yunlin, Taiwan.

Email: chuanyu@yuntech.edu.tw

Received November $13^{\text {th }}, 2012$; revised December $16^{\text {th }}, 2012$; accepted December $25^{\text {th }}, 2012$

\begin{abstract}
Recently medical cosmetic has attracted significant business opportunity. Micro cosmetic surgery usually involves invasive cosmetic procedures such as non-ablative laser procedure for skin rejuvenation. However, to select an appropriate treatment for skin relies on accurate preoperative evaluations. In this paper, an automatic facial skin defects detection and recognition method is proposed. The system first locates the facial region from the input image. Then, the shapes of faces were recognized using a contour descriptor. The facial features are extracted to define regions of interest and an image segment method is used to extract potential defect. A support-vector-machine-based classifier is then used to classify the potential defects into spots, acnes and normal skin. Experimental results demonstrate effectiveness of the proposed method.
\end{abstract}

Keywords: Medical Image Analysis; Texture Recognition; Skin Disease Identification; Spot and Acne Detection

\section{Introduction}

Recently medical cosmetology has a great development. It usually involves invasive cosmetic procedures or operation such as non-ablative laser procedure for skin rejuvenation and filling injection to relieve wrinkles [1,2]. Most conventional skin analysis instruments are contactbased. A physician has to visually inspect the target region and applies a contact test probe to magnify the target region for inspection. This contact testing procedure may be unsanitary. Non-contact inspections analyze patients' facial skin conditions by a camera directly. Doctors and patients are not necessary to be face to face. Furthermore, remote defect detection for consultation before cosmetology is possible.

VISIA is a widely used commercial instrument for skin analysis in cosmetic surgery. The skin condition is analyzed by multispectral images [3]. However, the cost is high and physicians should manual outline regions of interest (ROIs) during inspection.

With gradually higher resolution in digital cameras, many digital imaging methods have been proposed to analyze skin conditions [4-7]. These investigations applied various color quantization methods to distinguish whether the ROI is a spot or not. However, using pure color information to detect spots is difficult because shadow of facial organs (eyes, nose, mouth, or ears) may be misjudged as spots.

In addition, most skin analysis systems require manually outlining the regions of interest (ROIs) [3-5]. How- ever, manual ROI outlining is commonly known as a time-consuming and non-repeatable process.

In our prior work, an automatic facial skin defect detection system was proposed [7]. However, the facial view was restricted to front. Hence, a novel skin conditions evaluation system, which integrates a multi-view image acquisition device and automatic facial skin defect detection, is proposed in this paper. A facial features detection approach is applied to obtain positions of the facial features which are further applied to extract the ROI. A special color space and an adaptive threshold based on probability distribution are used to characterize potential defects from the ROI. Finally some significant texture features are extracted and applied to a specific designed classifier to classify potential defects into normal skin, spots and acnes.

The rest of this paper is organized as follows. Section 2 introduces details of the proposed skin defects detection and recognition system including definition of ROI, potential defect extraction and texture features used for classification. Section 3 demonstrates the experimental result. Conclusions and possible research topics in the future are given in Section 4.

\section{The Proposed System}

In this paper, an automatic facial skin defects detection and recognition system is proposed. When the facial images are captured by a high definition camera, the skin defects are detected and recognized automatically. Fig- 
ure 1 illustrates the processes of the proposed approach, includes face region detection, facial view detection, front/profile ROI extraction, potential defect extraction and classification. Details of these processes are described in the following subsections.

\subsection{Facial Region Detection}

A skin color detection method is applied to detect facial region. Soriano [8] proposed a skin locus in normalized color coordinate (NCC). This skin color model has performed well with images under widely varying conditions [9]. Therefore, the skin locus is adopted to detect skin-like region in this paper. A region filling method is applied to the largest connected region which is obtained by an 8 -adjacent connected component labeling algorithm. The largest skin region is regarded as the facial region [10]. The facial region detection result is shown in the Figure 2.

\subsection{Facial View Detection}

Before extraction of the ROI, the facial view needs to be determined. The shape of face can be used to distinguish between front and profile. Fourier descriptors have been widely used for representing boundary of a two-dimensional shape and it has the advantages of affine-invariant such as translation, rotation, and scaling [10]. In these descriptors, the centroid distance is used to represent the shape. The centroid distance $C d(t)$ is defined as follow:

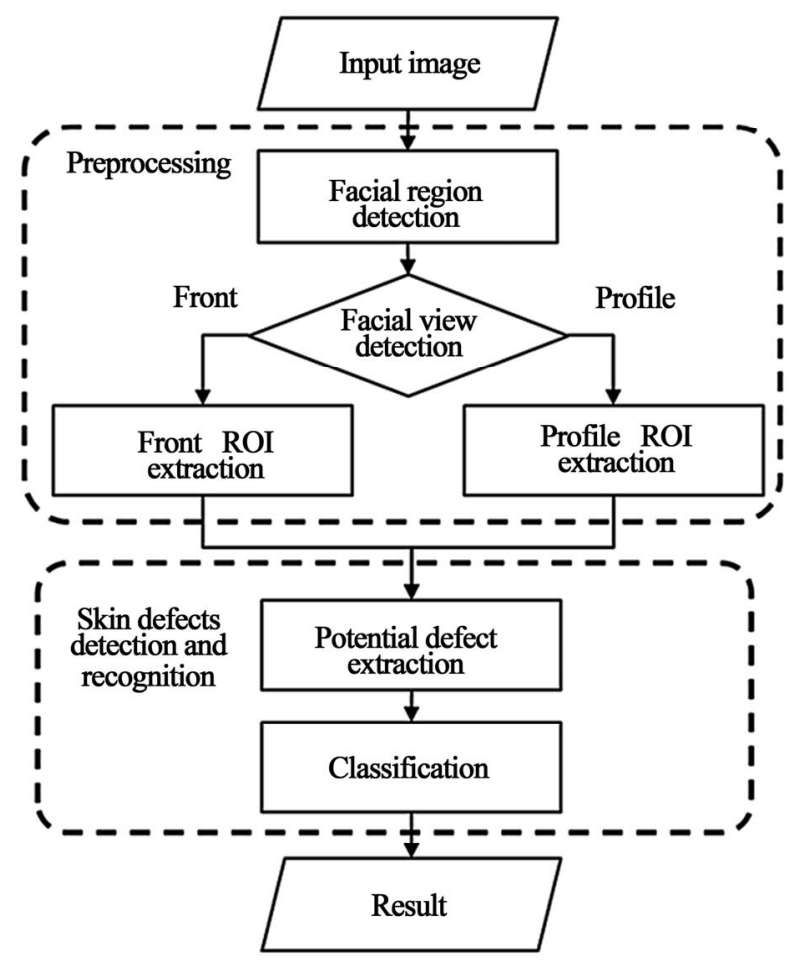

Figure 1. The flowchart of the proposed system.

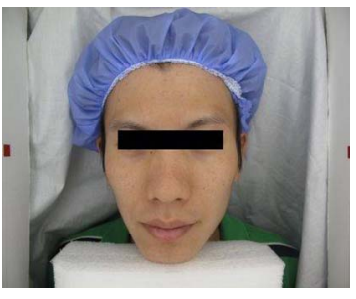

(a)

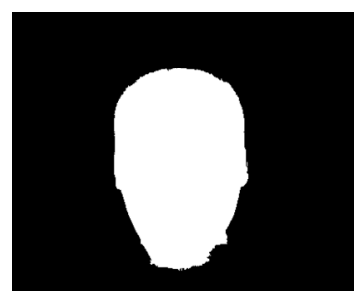

(b)
Figure 2. The facial region detection result: (a) Original image; (b) Detection result.

$$
\begin{gathered}
C d(t)=\sqrt{\left(x_{t}-x_{c}\right)^{2}+\left(y_{t}-y_{c}\right)^{2}}, t=0,1, \cdots, N-1 \\
x_{c}=\frac{1}{N} \sum_{t=0}^{N-1} x_{t}, y_{c}=\frac{1}{N} \sum_{t=0}^{N-1} y_{t}
\end{gathered}
$$

where $\left(x_{t}, y_{t}\right)$ is the $t$-th point on the face contour, $\left(x_{c}, y_{c}\right)$ is the centroid of the face contour, and $N$ is the number of sampling points. Fourier transform of $C d(t)$ is defined as follow:

$$
F D_{k}=\frac{1}{N} \sum_{t=0}^{N-1} C d(t) \exp \left(\frac{-j 2 \pi k t}{N}\right), k=0,1, \cdots, N-1
$$

Since $C d(t)$ is a real value, there are only $N / 2$ different frequencies in the Fourier transform, only half of $F D_{k}$ is needed to represent the face contour. Scale invariance is then obtained by dividing the magnitude values of the first half of $F D_{k}$ by DC component. The feature vector $\boldsymbol{F}$ is used as the input vector of support vector machine for training and testing.

$$
\boldsymbol{F}=\left[\frac{\left|F D_{1}\right|}{\left|F D_{0}\right|}, \frac{\left|F D_{2}\right|}{\left|F D_{0}\right|}, \cdots, \frac{\left|F D_{N / 2}\right|}{\left|F D_{0}\right|}\right]
$$

Because the Fourier descriptors have the attribution of affine-invariant, left and right profiles have the same result from the classifier. Hence, the direction of profile needs to be detected. The skin color distribution is used to distinguish between left and right profile. The numbers of skin pixels in the left profile and right profile are calculated respectively.

Figure 3 shows the statistics of skin pixels in the left profile and right profile, where $R$ and $L$ represent the number of skin pixel in the right profile and the left one, respectively. If $L$ is large than $R$, the direction of face is right, otherwise is left.

\subsection{ROI Extraction}

The facial features are further adopted to exclude undesired regions, including eyes, eyebrows, mouth and nostrils. The facial features are detected beforehand using empiricals information in $\mathrm{YCbCr}$ and $\mathrm{HSV}$ color spaces $[7,11]$. First, the pupils are detected under the $\mathrm{YCbCr}$ color space in the rough regions of both eyes. The rough 


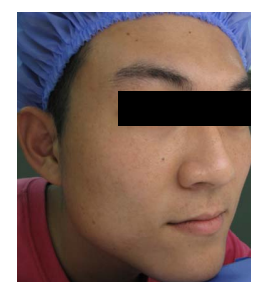

(a)

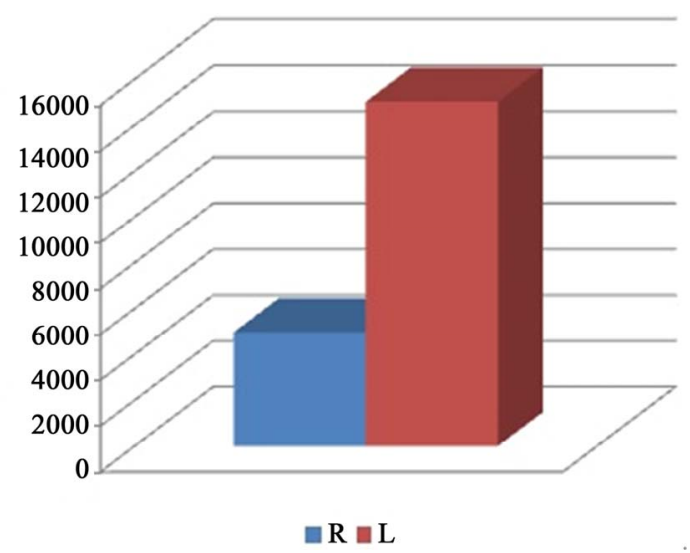

(c)

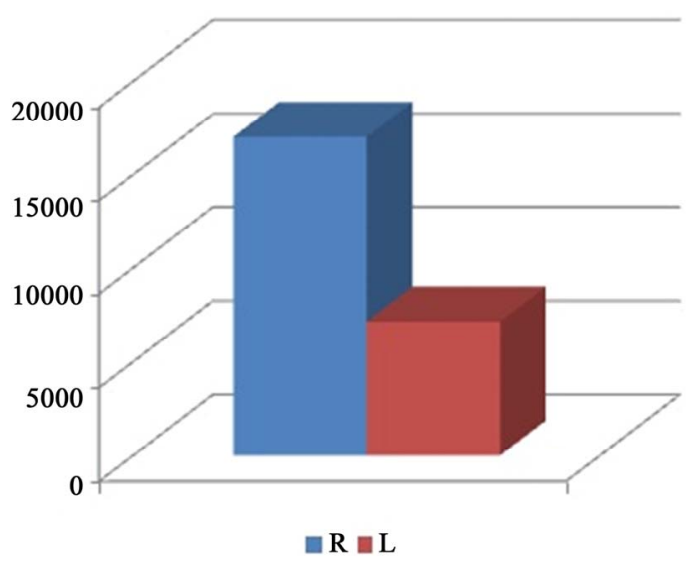

(d)

Figure 3. (a) Right profile; (b) Left profile; (c) The number of skin pixel in the right profile; (d) The number of skin pixel in the left profile.

regions of other facial features are located according to the position of pupils, as shown in the Figure 4. The eyes, eyebrows, mouth and nostrils are extracted by the Sobel, $\mathrm{HSV}$ color space, $\mathrm{YCbCr}$ color space and difference of Gaussian respectively in the corresponding region. The ROI is obtained by removing the facial features from the facial region. Figure 5 shows the results of ROI extraction in different view.

\subsection{Potential Defect Extraction}

In order to extract potential defects from the ROI, a segmentation method for potential defect detection is proposed. Because the surface of face is not a flat surface,

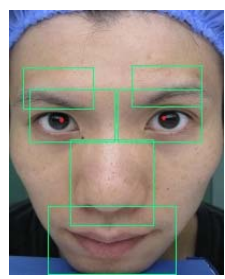

(a)

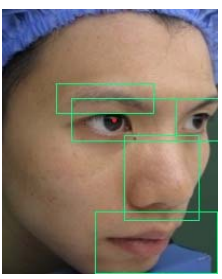

(b)
Figure 4. The rough regions of facial features: (a) Front; (b) Profile.
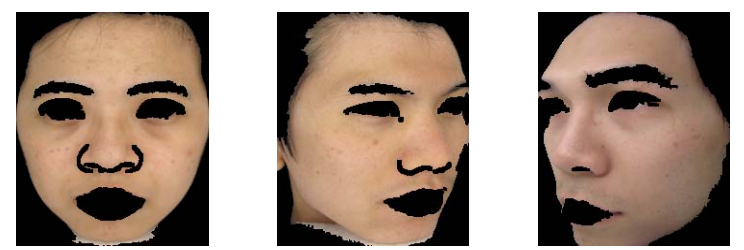

Figure 5. The results of ROI extraction.

skin color may varied in different positions on the same objects, even though the area focuses only on the forehead [7]. Therefore, the ROI image is divided into $n$ nonoverlap sub-images regularly. In this paper, the width and height of every sub-image are set to 100 .

The selection of color space is important for pattern recognition. $\mathrm{Cr}$ in $\mathrm{YCbCr}$ and Angle $\mathrm{A}$ has well human visual feedback for acnes and spots, respectively [12]. Therefore, $C r$-Angle $A$ color space is adopted to detect potential defects. The transformation functions are defined as follows:

$$
\begin{gathered}
C r=0.5 R-0.419 G-0.08 B+128 \\
\text { Angle } A=\cos ^{-1}\left(\frac{B}{L}\right)
\end{gathered}
$$

where

$$
L=\sqrt{R^{2}+G^{2}+B^{2}}
$$

and $R, G, B$ are three values corresponding to red, green and blue component in the original $R G B$ color space. The range of $C r$ value is $[0,255]$ and Angle $A$ is $[0,90]$.

Figure 6 shows an example of skin and defects distribution in $C r$-Angle $A$ space between two sub-images. We can find that the spot and acne have relatively high values in Angle $A$ and $C r$, respectively.

The center of skin color distribution $(C x, C y)$ is obtained by the maximum count of histogram in $\mathrm{Cr}$ and Angle $A$ respectively. $C x$ and $C y$ are defined as follows:

$$
\begin{gathered}
C x=\underset{k}{\arg \max } H_{k}\left(b_{C r}\right) \\
C y=\underset{k}{\arg \max } H_{k}\left(b_{\text {Angle } A}\right)
\end{gathered}
$$

where $H_{k}(\cdot)$ is the histogram in the $k$ level. The distance transform $D(x, y)$ between observational value and the center is defined as follow: 


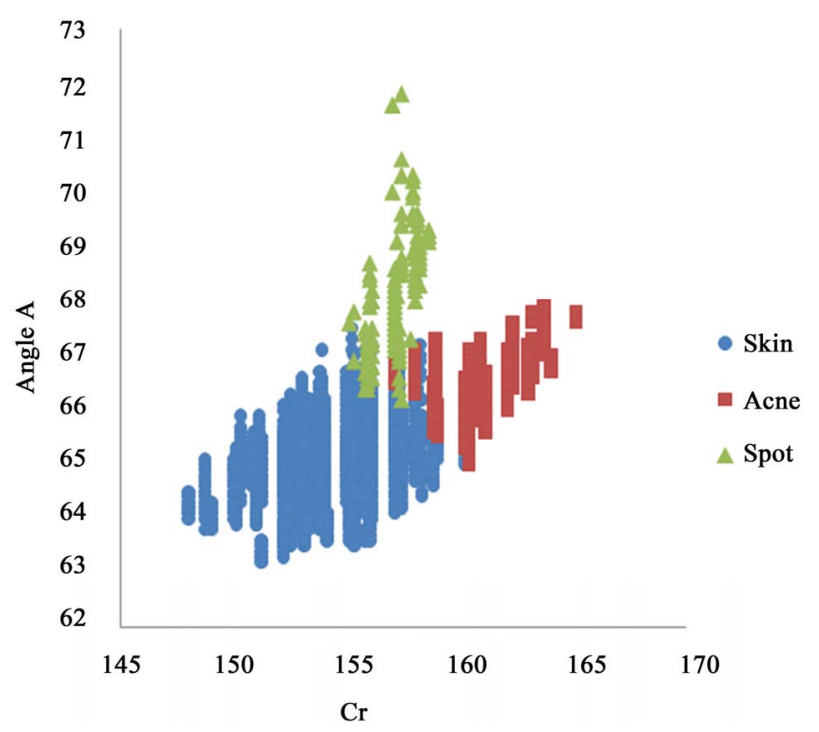

Figure 6. The example of skin and defects distribution in $C r$-Angle $A$ space.

$$
D(x, y)=\sqrt{\left[b_{C r}(x, y)-C x\right]^{2}+\left[b_{\text {AngleA }}(x, y)-C y\right]^{2}}
$$

If the distance transform is calculated, a threshold needs to be determined for distinguish potential defects and normal patterns. Then, the histogram thresholding based method is proposed. According to the observation, the probability density function of defect pixels occurrence in a sub-image is approximated by a Poisson distribution. Let the distances of $D(x, y)$ be represented in $L$ levels $[0,1, \cdots, L]$. The number of distances at level $k$ is denoted by $n_{k}$ and the total number of distances is $N=n_{1}$ $+n_{2}+\cdots+n_{L}$. The histogram of $D(x, y)$ is regarded as a probability density function $p_{k}$ :

$$
p_{k}=n_{k} / N, \quad \forall p_{k} \geq 0, \text { and } \sum_{k=0}^{L} p_{k}=1
$$

The curve fitting function $f_{k}(\lambda)$ which is shift by the Poisson is defined as follows:

$$
f_{k}(\lambda)=\frac{\lambda^{|k-m|+\lambda}}{(|k-m|+\lambda) !} e^{-\lambda}
$$

where

$$
m=\underset{k}{\arg \max }\left(p_{k}\right)
$$

Then, $f_{k}(\lambda)$ is scale to match $p_{k}$, Equation (12) is modified to Equation (14) which is defined as:

$$
f_{k}^{\prime}(\lambda)=f_{k}(\lambda) \times \frac{\operatorname{Max}\left(p_{k}\right)}{\operatorname{Max}\left(f_{k}(\lambda)\right)}
$$

where $\lambda=0,1, \cdots, L$. The fittest curve $p^{*}{ }_{k}$ is defined by finding a $\lambda$ such that the difference between $p_{k}$ and $f_{k}^{\prime}(\lambda)$ is minimum, and it is defined as follows:

$$
p_{k}^{*}=\underset{\lambda}{\arg \min }\left(\sum_{k}\left|f_{k}^{\prime}(\lambda)-p_{k}\right|\right)
$$

The threshold $t$ is set to the maximum value of the second order derivatives of $p^{*}{ }_{k}$. In other words, the pixel is regarded as foreground if the distance between observational pixel and the center in $C r$-Angle $A$ color space is larger than $t$; otherwise it is regarded as background. The threshold $t$ is defined as Equation (16).

$$
t=\underset{k}{\arg \max }\left[\nabla\left(p *_{k}\right)\right]
$$

where $\nabla(\cdot)$ is the second order derivative. The fittest curve and its second order derivative are shown in the Figure 7.

The potential defect is denoted by $S(x, y)$

$$
S(x, y)=\left\{\begin{array}{cc}
1 & \text { if } D(x, y)>t \\
0 & \text { otherwise }
\end{array}\right.
$$

Figure 8 shows the potential defect extraction method step by step. The potential defects can be obtained by a 4-adjacent connected component labeling algorithm.

\subsection{Classification}

\subsubsection{Feature Extraction}

To further classify the potential defects into normal patterns, acnes, and spots, the texture features calculated from co-occurrence matrix are used. The co-occurrence matrix is introduced by Haralick [13], which indicates probability of grey-level $i$ occurring in the neighborhood of grey-level $j$ at a distance $d$ and direction $\theta$. Fourteen co-occurrence matrix features including Contrast, Homogeneity, Mean, Variance, Energy, Entropy, Angular Second Moment, Correlation, Sum Average, Sum Entropy, Difference Average, Difference Variance and Difference Entropy are extracted [14]. In this paper, the distance was chosen as one pixel and four angles $\left(0^{\circ}, 45^{\circ}\right.$, $90^{\circ}, 135^{\circ}$ ) were selected.

To preserve spatial details, the texture features of the four directions are averaged. In addition to gray color, the color space $\mathrm{Cr}$ and Angle $A$ are also adopted. Accordingly, there are $14 \times 3$ GLCM features calculated from each potential defect. The averaged GLCM feature in the color space $C$ is denoted as

$$
t_{f}^{C}=\frac{t_{f, 0}^{C}+t_{f, 45}^{C}+t_{f, 90}^{C}+t_{f, 135}^{C}}{4}
$$

where $t_{f, \theta}^{C}$ represents the feature $f$ with angle $\theta$ in color $C$. A geometric feature roundness defined as Equation (17) is also adopted.

$$
\text { roundness }=\frac{E^{2}}{4 \pi A}
$$

where $E$ and $A$ are the perimeter and the area in a poten- 


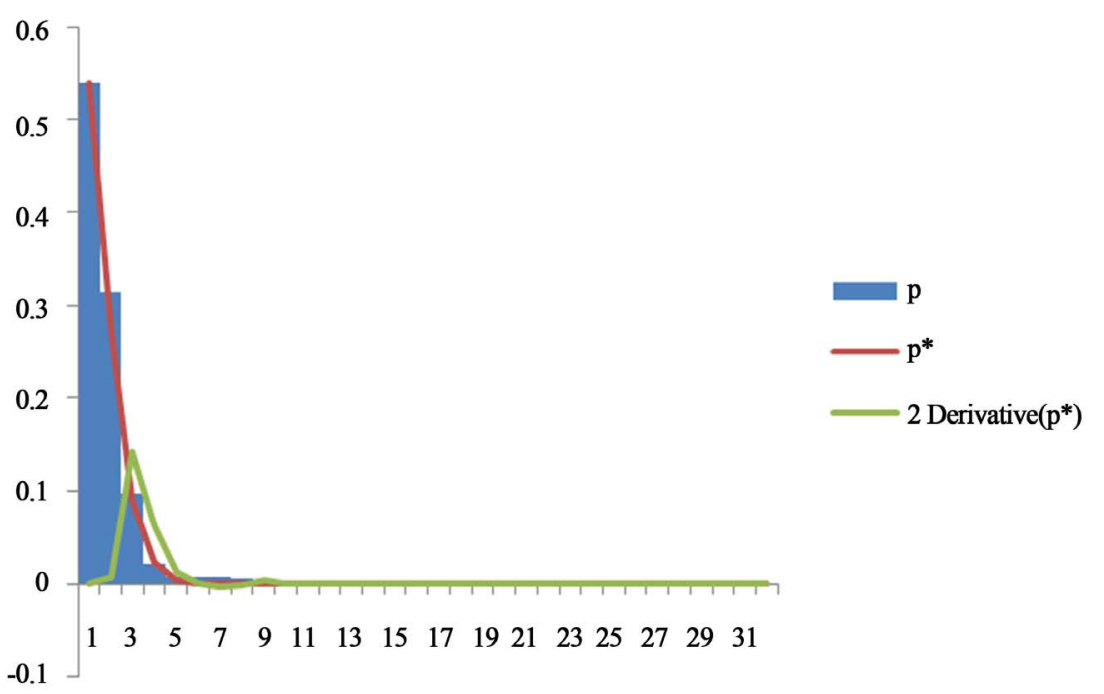

Figure 7. The result of threshold detection in a sub-image.

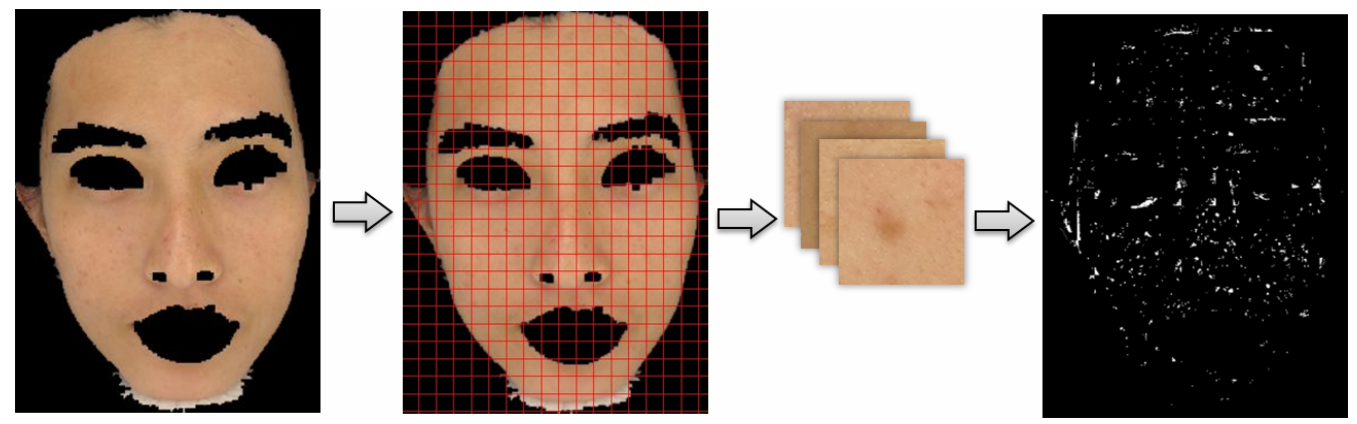

Figure 8. The potential defects extraction processing.

tial defect, respectively. Therefore, there are $42+1$ features in candidate features.

\subsubsection{Feature Selection}

In order to sift the most significant features, the sequential floating forward selection (SFFS) is adopted for feature selection [15]. This method consists of applying sequential forward selection (SFS) and sequential backward selection (SBS). By using the SFS and SBS repeatedly, it will converge when the defect recognition rate does not increase. In this paper, the cross validation rate is defined as the defect recognition rate which is implemented by LIBSVM [16]. There are 126 acnes, 134 spots and 134 normal patterns which are extracted manually from the database. Figure 9 shows some examples of the training patterns.

\subsubsection{Feature Extraction}

Support vector machine (SVM) is a popular and robust classifier in classification and regression analysis task. The SVM constructs a hyperplane in a high dimensional space which has the largest distance to the nearest training data points of any class. Figure 10 shows the struc- ture of the classifier. The classifier is utilized to classify the potential skin defects into normal patterns, acnes and spots, and the structure of classifier is a decision tree structure which consists of two SVMs. The SVM1 is used to classify defects and normal patterns from the potential defects. The other SVM2 is used to classify acnes and spots from the defects. Moreover, the SFFS algorithm is carried out individually in each stage.

\section{Experimental Results}

\subsection{Experiment Environment}

To reduce the influence of illumination, a special designed image acquisition device is created. Faces are captured by a high-resolution camera with resolution of $10 \mathrm{M}$ pixels.

Figure 11 shows the acquisition device. In this device, the acquisition parameters including camera parameters, light source, distances from subjects' face to the camera are all fixed. The camera is Cannon Power Shot G10. ISO speed is set to $1 / 60 \mathrm{sec}$. Focal length is set to automatic mode. Because the forehead is an important part in medical cosmetology, every subject is asked to move 


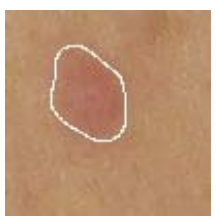

(a)

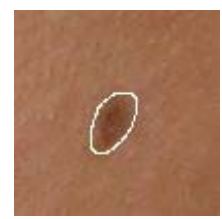

(b)

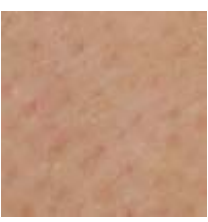

(c)
Figure 9. Some examples of three skin conditions: (a) Acnes; (b) Spots; (c) Normal patterns.

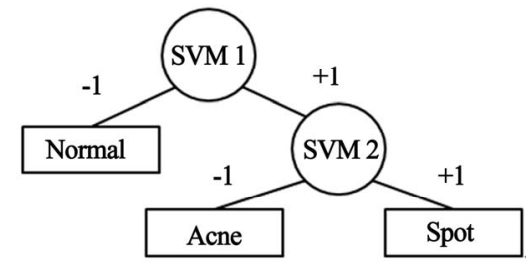

Figure 10. The structure of classifier.
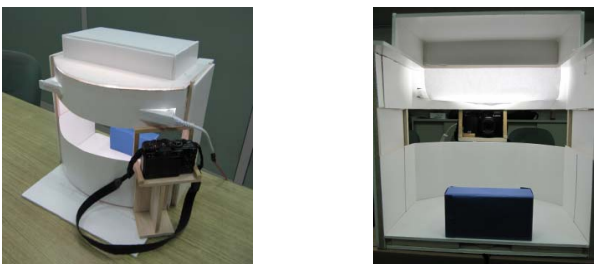

Figure 11. Image acquisition device.

their hair aside in order to show their forehead. Figure 12 shows some samples captured in this study. The system is implemented with Visual C\# and Python 2.5 on an Intel Core 2 Quad 2.66 GHz processor and $2 \mathrm{~GB}$ RAM platform.

\subsection{Experimental Database}

To demonstrate the capability of the proposed method, there are two face image database YUFH and YUFH2 are used. YUFH was created by Chang [7], and there are 93 face images taken from 3 females and 26 males in 3 views (front, left profile and right profile). YUFH2 is collected by our image acquisition device. There are 54 face images taken from 3 females and 15 males in 3 views.

\subsection{Face View Classification Result}

To demonstrate the capability of the face view classification, the two databases are used. In each database, a half of subjects are used for training, rest of subjects are used for testing. The Confusion Matrix for face view classification result in the database YUFH and YUFH2 are shown in Tables $\mathbf{1}$ and $\mathbf{2}$ respectively.

\subsection{Feature Selection Result}

The result of feature selection by SFFS for SVM1 is shown

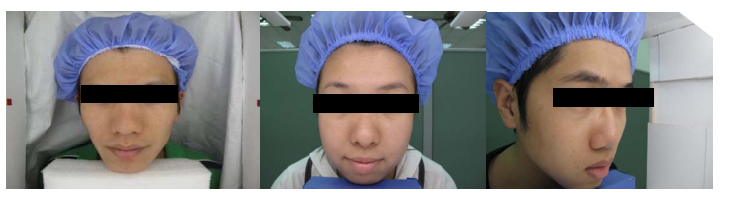

(a)

(b)

(c)

Figure 12. Some examples in the database: (a) YUFH; (b), (c) YUFH2.

Table 1. The confusion matrix for face view classification in YUFH.

\begin{tabular}{cccc}
\hline Result & Front & Profile & Accuracy \\
\hline Front & 12 & 0 & 100.0 \\
Profile & 0 & 24 & 100.0 \\
& Average accuracy & & 100.0 \\
\hline
\end{tabular}

Table 2. The confusion matrix for face view classification in YUFH2.

\begin{tabular}{cccc}
\hline Result & Front & Profile & Accuracy \\
\hline Front & 8 & 1 & 88.9 \\
Profile & 0 & 18 & 100.0 \\
& Average accuracy & & 96.2 \\
\hline
\end{tabular}

in Table 3. For SVM2, the result of feature selection by SFFS is shown in Table 4.

\subsection{Defect Detection Result}

To quantify the performance of the proposed approach, three standardized measurements are adopted: Accuracy, Sensitivity and Specificity. They are defined respectively as follows:

$$
\begin{gathered}
\text { Accuracy }=\frac{R_{T P}+R_{T N}}{R_{P}+R_{N}} \\
\text { Sensitivity }=\frac{R_{T P}}{R_{P}} \\
\text { Specificity }=\frac{R_{T N}}{R_{N}}
\end{gathered}
$$

where $R_{P}$ is the total number of defect regions and $R_{N}$ is the total number of normal regions. $R_{T P}$ is the number of regions in the actual defect region and is classified as defect by the proposed system and $R_{T N}$ is the number of regions in the actual normal region and classified as normal by the system. In this paper, the $R_{N}$ is defined as $R_{P}$ subtracted from the total number of potential defect regions.

Table 5 shows the evaluation results of the proposed approach in the YUFH database. There are 93 face im- 
Table 3.The result of SFFS for SVM1.

\begin{tabular}{cc}
\hline Feature & Color Space \\
\hline Contrast & AngleA \\
Correlation & AngleA \\
Difference Variance & AngleA \\
Variance & Cr \\
Sum Variance & Cr \\
Homogeneity & Gray \\
Variance & Gray \\
Entropy & Gray \\
Sum Average & Gray \\
Difference Average & Gray \\
Mean & Gray \\
Roundness & - \\
\hline
\end{tabular}

Table 4. The result of SFFS for SVM2.

\begin{tabular}{cc}
\hline Feature & Color Space \\
\hline Contrast & AngleA \\
Homogeneity & AngleA \\
Entropy & AngleA \\
Angular Second Moment & AngleA \\
Correlation & AngleA \\
Sum Entropy & AngleA \\
Difference Average & AngleA \\
Difference Variance & AngleA \\
Difference Entropy & AngleA \\
Correlation & Cr \\
Contrast & Gray \\
Variance & Gray \\
Energy & Gray \\
Angular Second Moment & Gray \\
Difference Average & Gray \\
Difference Variance & Gray \\
\hline
\end{tabular}

Table 5. The evaluation result of the proposed approach in YUFH.

\begin{tabular}{cccc}
\hline & Accuracy & Sensitivity & Specificity \\
\hline Spot & $96.94 \%$ & $76.56 \%$ & $97.56 \%$ \\
Acne & $98.22 \%$ & $64.52 \%$ & $98.41 \%$ \\
\hline
\end{tabular}

ages including front and profile. Table 6 shows the evaluation results of the proposed approach in the YUFH2 database. There are 54 face images including front and profile. In these tables, the Sensitivity measurement in acne detection is relatively low than the others. The reason is that acnes have various types and stages in clinical treatment.

Figure 13 shows the result of defects detection, in which the spots and acnes are outlined in blue and red, respectively.

We compared the performance of the proposed approach for spots with Chang [6] and Chang [7]. Table 7
Table 6. The evaluation result of the proposed approach in YUFH2.

\begin{tabular}{cccc}
\hline & Accuracy & Sensitivity & Specificity \\
\hline Spot & $93.63 \%$ & $75.62 \%$ & $94.59 \%$ \\
Acne & $99.68 \%$ & $69.73 \%$ & $99.90 \%$ \\
\hline
\end{tabular}

Table 7. Comparison of the proposed approach and others.

\begin{tabular}{cccc}
\hline & Accuracy & Sensitivity & Specificity \\
\hline Proposed method & $99.40 \%$ & $80.91 \%$ & $99.42 \%$ \\
Chang [6] & $98.76 \%$ & $54.34 \%$ & $98.81 \%$ \\
Chang [7] & $99.22 \%$ & $63.24 \%$ & $99.25 \%$ \\
\hline
\end{tabular}

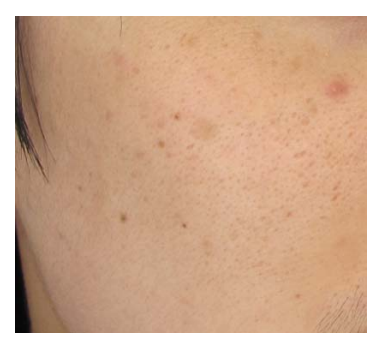

(a)

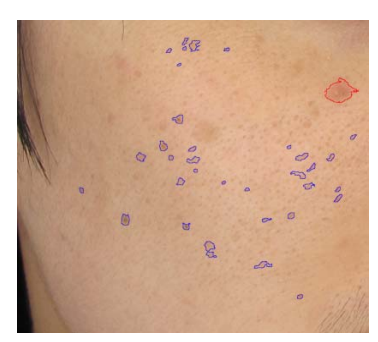

(b)
Figure 13. (a) Original image; (b) Result of defects detection.

shows the results obtained using the proposed approach and [6,7]. There are 97 images including 32 males and 4 females from both YUFH and YUFH2. From this table, one can see accuracies of the proposed approach for spots detection are higher than those of [6] and [7].

\section{Conclusions and Future Directions}

Skin analysis is one of the most important procedures before medical cosmetology. In this paper, an automatic facial skin defects detection and recognition system is proposed. Different from other methods which have to manually outline the ROIs in a face image, the proposed approach obtains the ROI automatically in both front and profile views.

The proposed system first locates the facial region from the input image. The facial features and skin color were used to locate the ROI. An approximate Poisson distribution is used to define a suitable threshold for extracting potential defects. Then, the SFFS is adopted to select significant features for defects classification. Finally, a decision tree classifier consists of two SVMs is applied to classify the potential defects into normal patterns, spots and acnes. Experimental results show that the proposed method can detect facial skin defect and recognize the lesion effectively.

In the future work, more images of subjects in different ages will be collected and tested. The accuracy of the current system can increase by attempting more features. 


\section{Acknowledgements}

This work was supported by the National Science Council Taiwan, under Grants NSC 98-2220-E-224-02.

\section{REFERENCES}

[1] J. Goldberg, "Photodynamic Therapy in Skin Rejuvenation," Clinics in Dermatology, Vol. 26, No. 6, 2008, pp. 608-613. doi:10.1016/j.clindermatol.2007.09.009

[2] W. Buck Ii, M. Alam and J. Y. S. Kim, "Injectable Fillers for Facial Rejuvenation: A Review," Journal of Plastic, Reconstructive \& Aesthetic Surgery, Vol. 62, No. 1, 2009, pp. 11-18. doi:10.1016/j.bjps.2008.06.036

[3] Digitale Photographie GmbH, "VISIA Complexion Analysis," 2004.

http://www.visia-complexion-analysis.com/visia-complex ion-analysis.asp

[4] S. N. Yeh, C. C. Chen and H. H. Wu, "Design and Implementation of a Facial Image Acquisition and Analysis System," Proceeding of Workshop on Consumer Electronics and Signal Processing, Yulin, 17-18 November 2009.

[5] H. C. Lee, W. J. Kuo and H. H. Huang, "Research on the Features of Human Skin Appearance by Image Processing," Master Thesis, Yuan Ze University, Jhongi City, 2006.

[6] T. R. Chang and C. Y. Huang, "Skin Condition Detection Based on Image Processing Techniques," Proceeding of Conference on Information Technology and Applications in Outlying Island, Penghu, May 2010.

[7] C. Y. Chang, S. C. Li, P. C. Chung and J. Y. Kuo, "Automatic Facial Skin Defect Detection System," Proceeding of 2010 International Conference on Broadband, Wireless Computing, Communication and Applications, 4-6 November 2010, pp. 527-532. doi:10.1109/BWCCA.2010.126

[8] M. Soriano, B. Martinkauppi, S. Huovinen and M. Laak- sonen, "Using The Skin Locus to Cope with Changing Illumination Conditions in Color-Based Face Tracking," IEEE Nordic Signal Processing Symposium, Vol. 38, 2000, pp. 383-386.

[9] M. P. Hadid and B. Martinkauppi, "Color-Based Face Detection Using Skin Locus Model and Hierarchical Filtering," 16th International Conference on Pattern Recognition Proceedings, Vol. 4, 2002, pp. 196-200.

[10] C. Y. Chang, C. W. Chang and J. S. Li, "Multi-View Facial Feature Extraction," Information-An International Interdisciplinary Journal, Vol. 16, 2013, pp. 191-204.

[11] C. Y. Chang, Y. C. Huang and P. C. Chung, "Personalized Facial Expression Recognition for Indoor Space," Proceeding of 22nd Conference on Computer Vision, Graphics and Image Processing Conference, Nan-Tou, 2325 August 2009.

[12] S. E. Umbaugh, R. H. Moss and W. V. Stoecker, "Automatic Color Segmentation of Images with Application to Detection of Variegated Coloring in Skin Tumors," IEEE Engineering in Medicine and Biology Magazine, Vol. 8, No. 4, 1989, pp. 43-50. doi:10.1109/51.45955

[13] R. M. Haralick, "Statistical and Structural Approaches to Texture," Proceedings of IEEE, Vol. 67, No. 5, 1979, pp. 786-804. doi:10.1109/PROC.1979.11328

[14] Y. Chang, S. J. Chen and M. F. Tsai, "Application of Support-Vector-Machine-Based Method for Feature Selection and Classification of Thyroid Nodules in Ultrasound Images," Pattern Recognition, Vol. 43, No. 10, 2010, pp. 3494-3506. doi:10.1016/j.patcog.2010.04.023

[15] Guyon, "Feature Extraction: Foundations and Applications," Springer, Berlin, 2006.

[16] C. C. Chang and C. J. Lin, "LIBSVM: A Library for Support Vector Machines," 2001. http://www.csie.ntu.edu.tw/ cjlin/libsvm 